\title{
Pressure gradient effects on wake flow instabilities behind isolated roughness elements on re-entry capsules
}

\author{
Alexander Theiss, Sascha Leyh and Stefan Hein \\ DLR, Institute of Aerodynamics and Flow Technology \\ Bunsenstr. 10, 37073 Göttingen, Germany \\ alexander.theiss@dlr.de·stefan.hein@dlr.de
}

\begin{abstract}
Laminar-turbulent transition caused by modal disturbance growth in the wake flow of isolated roughness elements on blunt re-entry capsules is studied numerically at typical cold hypersonic wind tunnel conditions. Two fundamentally different heat shield shapes are considered. On the sphere-cone forebody the wake flow of the roughness is exposed to an adverse pressure gradient, whereas the spherical heat shield exhibits a strongly favourable pressure gradient. The pressure gradient effects on the development of the stationary wake flow and its modal instability characteristics are discussed for various heights and diameters of the cylindrical roughness element. Regions of increased shear develop in its wake which persist longer in the adverse pressure gradient case. Consequently, the results of spatial two-dimensional eigenvalue analyses reveal that the unstable wake flow region extends much further downstream and the wake mode instabilities are considerably more amplified. The disturbance kinetic energy production terms are used to assess the contributions of the different shear-layer regions to the mode growth and its dependence on the pressure gradient.
\end{abstract}

\section{Introduction}

Accurate prediction of boundary layer transition on space re-entry vehicles is critical to design improvements of the thermal protection system (TPS). Entering aft-end first from space into a planet's atmosphere, the heat shield of a reentry capsule has to withstand very high heat loads. Due to a lack of appreciation and understanding of the physics of transition on such blunt vehicles, the design philosophy so far assumed a fully turbulent flow in flight at all times leading to an over-dimensioning of the TPS, associated with cutbacks on the feasible payload. One possibility to carry more cargo with the same overall weight is to reduce the mass of the heat shield while keeping the level of safety constant. This can be achieved by applying more advanced (lighter) TPS materials or by taking all relevant flow regimes into account. For a potential TPS weight reduction, it is absolutely essential to correctly estimate the expected heat transfer while taking all laminar flow regions and the transition location into consideration.

The boundary layer on capsules with a spherical forebody does not feature any amplified modal disturbances at hypersonic speeds and typical ground-based wind tunnel conditions. ${ }^{35}$ The strong favorable pressure gradient keeps the first mode stable and amplified second-mode disturbances do not occur due to subsonic to transonic post-shock boundary layer edge Mach numbers. Moreover, the spherical shape does not promote sufficient crossflow and its convex nature prevents Görtler instabilities. In contrast, capsules with a sphere-cone shape exhibit amplified first-mode and crossflow instabilities at similar freestream conditions (depending on the cone angle). ${ }^{8,38}$

There exist several sources of instability and mechanisms that accelerate the laminar-turbulent transition process, which are specific to hypersonic flows over blunt capsules. One of them is out-gassing from an ablative heat shield. $\mathrm{Li}$ et al. $^{24}$ demonstrated the destabilizing effect of ablation-induced out-gassing on a boundary layer flow over a hemispherical capsule which otherwise did not support amplification of instability modes. Ablation of the heat shield can also cause the formation of a distributed surface micro-roughness pattern, which in turn can lead to an earlier boundary-layer transition compared to the smooth wall case. Experimental investigations on premature boundary layer transition due to distributed roughness effects on re-entry capsules have been conducted by Grossir et al. ${ }^{15}$ (EXPERT vehicle at Mach 14), Hollis ${ }^{18}$ (sphere-cone and hemisphere geometries at Mach 6) and Leidy et al. ${ }^{22}$ (Orion Crew Exploration Vehicle (CEV) at Mach 6). A different common type of roughness for realistic re-entry vehicles are discrete roughness elements, such as attachment-point cavities, compression pads, gaps or steps between heat shield 
tiles or unevenly ablated TPS material. A single protuberance element can also trigger the laminar-turbulent boundary transition in its wake or even cause bypass transition if the roughness height/cavity depth is sufficiently large, though it is not as effective as distributed roughnesses with the same height (see Reda ${ }^{31}$ and Reda et al. ${ }^{32}$ ).

Amar et al. ${ }^{2}$ and Berger ${ }^{4}$ demonstrated the effect of isolated protuberances on the laminar to turbulent transition on the Orion CEV capsule in wind tunnel experiments at low enthalpy conditions. Tripping of the boundary layer was achieved with diamond-shaped discrete roughness elements to evaluate the effectiveness of the trips (Amar et $\mathrm{al}^{2}$ ) and to obtain turbulent forebody heating data for comparison with computational predictions $\left(\right.$ Berger $\left.^{4}\right)$. Tanno et al. ${ }^{34}$ and Marineau et al. ${ }^{27}$ investigated the boundary layer transition on an Apollo-shaped capsule at high enthalpy conditions and also used discrete roughnesses to induce transition on the leeside of the model. An overshoot in heat transfer downstream of the roughness followed by a progressive relaxation to computed turbulent values was observed by Marineau et al., ${ }^{27}$ whereby the amplitude of the overshoot increased with protuberance height. Direct numerical simulations (DNS) of Chang et al. ${ }^{9}$ for surface irregularities (protuberance and cavity) on the CEV at wind tunnel conditions revealed that a roughness sticking out of the surface is more effective to trigger transition than a cavity at identical roughness Reynolds number $\left(R e_{k k}\right)$ and Reynolds number values. An extensive summary of experimental work, which focuses on roughness related transition in hypersonic boundary layers on blunt bodies is given by Reda ${ }^{31}$ and Schneider. ${ }^{33}$

The mechanisms leading to transition behind a three-dimensional roughness are only partly understood, but work on mainly super- and hypersonic roughness-induced transition (carefully conducted experiments, ${ }^{20,40}$ extensive DNS simulations ${ }^{5,13,19,28,39}$ and results from global, ${ }^{26}$ two-dimensional linear eigenvalue (LST-2D) ${ }^{10,12,14,36}$ and threedimensional parabolized (PSE-3D) $)^{37,39}$ instability analyses) have considerably increased the knowledge in the recent years. However, most investigations have been performed on flat plates with a zero pressure gradient (ZPG) and a detailed study on the influence of different pressure gradients on roughness wake flow instabilities is still missing. Theiss and Hein ${ }^{36}$ and Theiss et al. ${ }^{37}$ used LST-2D to characterize the most amplified wake modes behind different roughness elements in the symmetry plane of an Apollo-shaped capsule model and showed that the zone of modal amplification was much shorter in comparison to the length scales computed by Choudhari et al. ${ }^{10,12}$ for a roughness element with similar $R e_{k k}$ values in a flat plate flow. The authors speculated that the deviations to the ZPG result origin in the strong favorable pressure gradient (FPG) on the capsule forebody. Recently, Plogmann et al. ${ }^{29}$ experimentally investigated the effect of different pressure gradients on the mode amplitudes behind a cylindrical roughness element in the incompressible flow regime. The authors reveal that in the near wake of the roughness the amplitude of the leading mode is very weakly dependent on the pressure gradient. However, the amplitude in the favorable pressure gradient case is slightly lower compared with the zero and adverse pressure (APG) case. For the far wake region, the authors report that the leading mode is damped in the FPG case, whereas in the ZPG and APG case the amplitude remains at a nearly constant level before being slightly damped. ${ }^{29}$ A similar observation is also mentioned in the work by Chang et al. ${ }^{9}$

The present paper provides a continuation of our previous work (see Refs. 36, 37) on wake flow instabilities behind an isolated roughness element on the forebody of a blunt generic re-entry capsule at cold hypersonic flow conditions. The intent of this paper is to clarify the effect of different pressure gradients on discrete roughness-induced transition. This work focuses on the instability properties of the streaky wake flow behind a cylindrical roughness element located in the adverse pressure gradient region of a $50^{\circ}$ sphere-cone capsule. Results from laminar basic flow computations for several roughness heights and diameters at similar roughness Reynolds numbers, $R e_{k k}$, and identical slenderness ratios, $d / k$, as in our previous studies (Apollo-shaped capsule with a strong favorable pressure gradient), will be presented and the wake flow instability characteristics will be analyzed with the help of spatial two-dimensional instability theory. The influence of different pressure gradients (spherical capsule forebody versus sphere-cone shape) on the various types of wake modes, the length of the amplified wake flow region and the maximum cumulative amplification ratios ( $\mathrm{N}$-factors) will be discussed.

\section{Numerical methods}

The first part of this section provides an overview of the investigated capsule configurations and the applied freestream conditions for the numerical simulations. Details about the laminar basic flow computations are subsequently given for the sphere-cone capsule without and with an isolated roughness element located on the conical part of the capsule forebody. Subsection 2.3 explains the two-dimensional linear stability theory used to characterize the wake flow instabilities behind the isolated roughness element. 

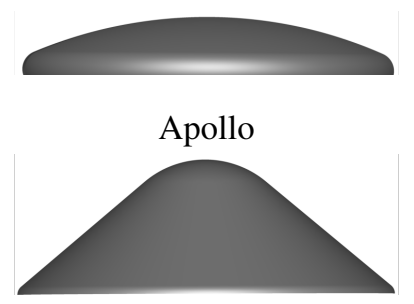

MSL, $\theta=50^{\circ}$

Figure 1: Investigated capsule forebody geometries.
Table 1: Overview of investigated freestream conditions.

\begin{tabular}{ccccccc}
\hline Model & $\mathrm{AOA}\left[{ }^{\circ}\right]$ & $R e_{\infty}[1 / \mathrm{m}]$ & $\mathrm{Ma}$ & $p_{\infty}[\mathrm{Pa}]$ & $T_{\infty}[\mathrm{K}]$ & $T_{\text {wall }}[\mathrm{K}]$ \\
\hline $50^{\circ}$-MSL & 16 & $24.05 \times 10^{6}$ & 6.02 & 2090 & 63.8 & 300 \\
Apollo & 24 & $10.00 \times 10^{6}$ & 5.9 & 764.9 & 59.0 & 295 \\
\hline
\end{tabular}

\subsection{Capsule models and freestream conditions}

The first model studied is an Apollo-shaped capsule with a base diameter of $D=170 \mathrm{~mm}$. This geometry features a strong favorable pressure gradient on the spherical forebody. The second configuration with an APG is a variant of the Mars Science Laboratory vehicle with a sphere-cone forebody and a base diameter of $D=152.4 \mathrm{~mm}\left(50^{\circ}\right.$-MSL). An overview of the investigated forebody shapes is given in Fig. 1. The freestream values for the laminar basic flow computations were determined from wind tunnel conditions to match the experiments of Ali et al. ${ }^{1}$ for the Apollo capsule and Hollis et al. ${ }^{17}$ for the MSL configuration and are summarized in Table 1.

\subsection{Laminar basic flow computation}

This subsection outlines the meshing strategies and the numerical tools used to simulate a generic re-entry capsule at hypersonic flow conditions without and with an isolated cylindrical roughness element on its forebody. The focus here is on the MSL configuration and details about the basic flow computations for the Apollo-shaped model can be found in Refs. 35, 36. Furthermore, section 2.2.2 includes considerations on the optimal roughness position to work out the effects of an APG on wake flow instabilities.

\subsubsection{Smooth capsule configuration}

The unperturbed boundary layer flow on the inclined MSL surface is computed on block-structured grids using a second-order accurate AUSMDV upwind scheme, as implemented in DLR's finite-volume compressible Navier-Stokes flow solver FLOWer. ${ }^{30}$ A grid convergence study was performed (details can be found in Ref. 25) and the singularityfree, shock-adapted mesh has about $12.1 \times 10^{6}$ points with at least 100 cells in the boundary layer. Due to the short operating times of the wind tunnel, a uniform isothermal wall boundary condition was imposed (see Table 1).

\subsubsection{Capsule with isolated roughness element}

A cylindrical roughness element of various heights and diameters is placed in the leeward symmetry plane on the conical part of the capsule forebody. Only half of the element was simulated in the truncated computational domain (depicted in Fig. 2), exploiting the azimuthal symmetry of the flow field. The approach of using a smaller domain for the simulations with roughness element substantially decreased the required number of points for the laminar basic flow computations. As an additional benefit, the truncated domain is located below the bow shock, so a 2 nd-order central scheme was chosen for the spatial discretization in order to speed up the simulations.

Figure 3 depicts the structured grid used to discretize the flow field around the sharp edged roughness elements (only every 4th grid point is shown). To maintain a reasonable computational effort, clustering of grid points towards the element in all direction was applied and the mesh consists of approximate $150 \times 10^{6}$ points with about 1100,260 , and 300 points behind the roughness in streamwise, spanwise, and wall-normal direction, respectively. The mesh topology, as well as the number of grid points, is identical to the one of our previous studies (see Refs. 36, 37). Nevertheless, grid convergence was also checked. ${ }^{23}$

The cylindrical roughness element is placed on the conical part of the capsule at $x_{2} / R=0.45$. As indicated in Fig. 4, at this position the protuberance is in the APG region and exhibits an adequate margin towards both the FPG region on the spherical nose part and the downstream position where first-mode instabilities start to grow (see Refs. 8, 25,38). The objective of this work is to exclusively study convective wake flow instabilities and to exclude hybrid modes with significant fluctuations in the wake region as well as in the outer region. The solid lines in Fig. 4 


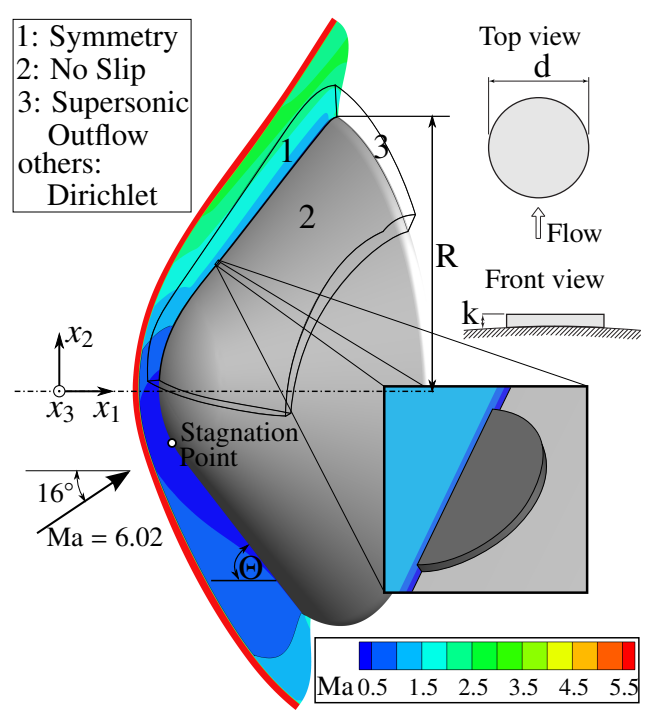

Figure 2: Scheme of the truncated domain used for the roughness wake flow simulations with the specified boundary conditions.

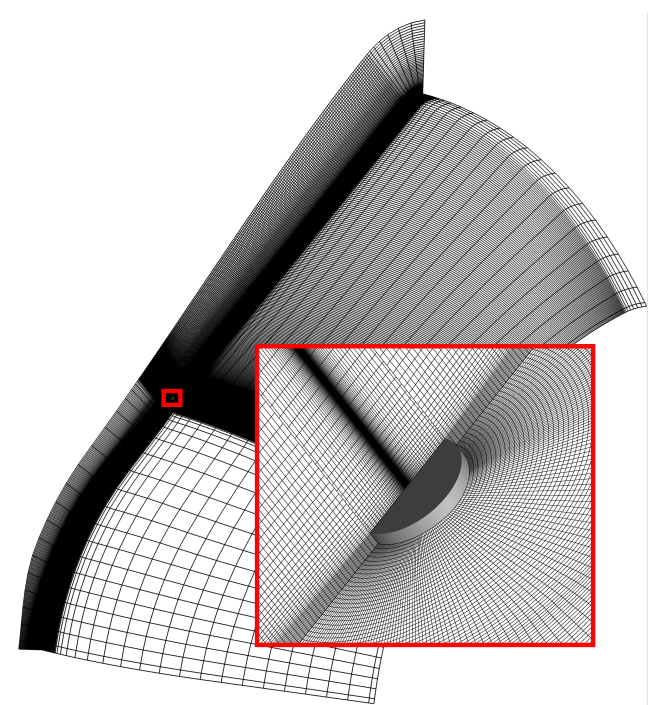

Figure 3: Detail of the computational grid with close-up near the roughness position. Only every 4th point is shown for clarity.

show the evolution of the roughness height $k$ along the capsule surface at constant roughness Reynolds numbers (the values that are used in this work). The roughness Reynolds number, $R_{k k}$, is based on the roughness height $k$ and the flow values of the unperturbed basic flow at that height. For the chosen roughness position, it is assumed that a shock will not occur above the cylinder, due to a sufficient vertical margin towards the sonic roughness Mach number $M a_{k}$, which is also defined with the values of the unperturbed basic flow at the roughness edge.

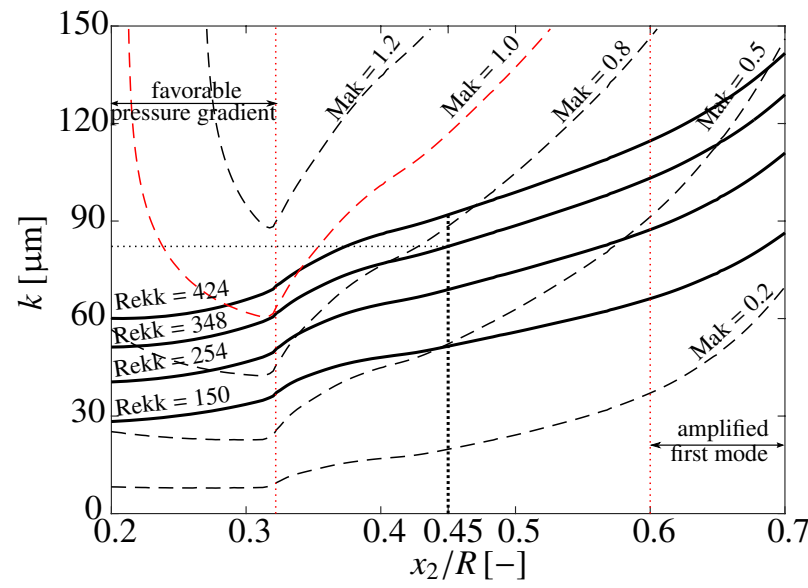

Figure 4: Evolution of the resulting roughness height along the $50^{\circ}$-MSL capusle centerline at fixed roughness Reynolds number. The vertical dashed line at $x_{2} / R$ marks the roughness position.
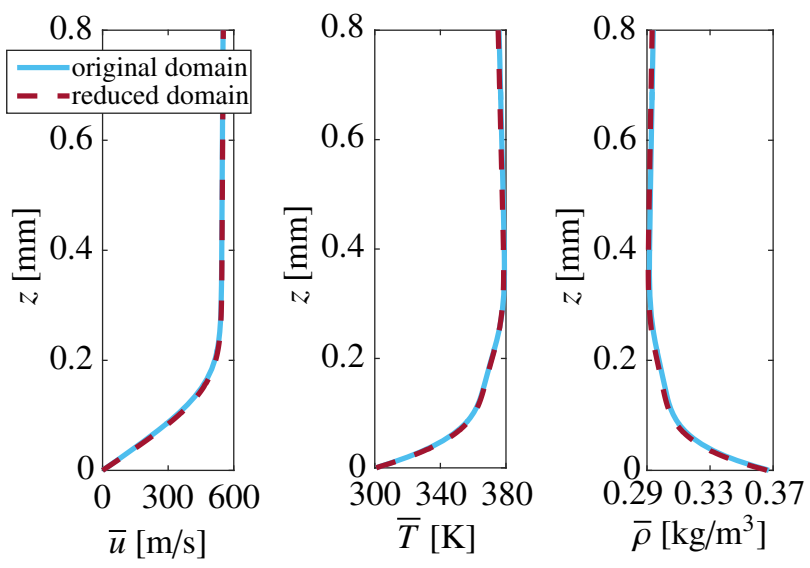

Figure 5: Comparison of boundary layer profiles for the smooth sphere-cone capsule computed in the original and in a reduced domain, $x_{2} / R=0.45$.

The numerical boundary conditions imposed are those as depicted in Fig. 2. Converged basic flow data without the protuberance element on the capsule are specified on all sides of the computational domain via a Dirichlet boundary condition, except for the wall, the outflow region, and the capsule zero meridian, where the no-slip, supersonic outflow, and symmetry conditions are set, respectively. The suitability of the Dirichlet boundary condition was checked by simulating the reduced domain without disturbance element. The boundary layer profiles of the converged flow within the smaller domain show a very good agreement with the original solution as depicted in Fig. 5 for the roughness location as an example. 


\subsection{Two-dimensional linear stability theory - LST-2D}

The wake flow behind an isolated roughness element has a strong inhomogeneous character in both wall-normal and spanwise direction. Thus, the conventional linear stability analysis, which is based on the assumption of inhomogeneity in just one spatial direction, is not appropriate to analyze the stability characteristics of wake flows and a 2D eigenvalue problem is solved instead.

The LST-2D is based on the non-dimensional version of the compressible Navier-Stokes equation and analogue to the ansatz of classical linear stability theory. All flow quantities $\mathbf{q}=[\rho, u, v, w, T]^{T}$ are split into their steady primary state $\overline{\mathbf{q}}$ and unsteady perturbation part $\tilde{\mathbf{q}}$ :

$$
\mathbf{q}(x, y, z, t)=\overline{\mathbf{q}}(y, z)+\epsilon \tilde{\mathbf{q}}(x, y, z, t), \quad \epsilon \ll 1,
$$

whereby the primary state $\overline{\mathbf{q}}$ and the total flow field $\mathbf{q}$ are assumed to satisfy the Navier-Stokes equations. However, two spatial inhomogeneous directions are simultaneously resolved on a plane (wall normal $z$ and spanwise $y$ direction), whereas spatial homogeneity in the third direction is assumed (streamwise $x$ direction). The infinitesimal threedimensional perturbations $\epsilon \tilde{\mathbf{q}}$ are inhomogeneous in $z$ and $y$ and periodic in $x$. After applying the modal perturbation ansatz $\tilde{\mathbf{q}}$, one may write

$$
\tilde{\mathbf{q}}(x, y, z, t)=\hat{\mathbf{q}}(y, z) \Theta(x, t)+c . c ., \Theta=\exp [i(\alpha x-\omega t)],
$$

with $\hat{\mathbf{q}}$ defining the vector of the two-dimensional complex amplitude functions in a plane perpendicular to the $x$-axis, $\Theta$ representing the complex phase function and c.c. denoting the complex conjugate. The LST-2D equations are obtained by substituting the perturbed state (Eqn. 1) into the Navier-Stokes equation, assuming local parallel flow, subtracting out the $O(1)$ basic flow terms and neglecting terms at $\left(O\left(\epsilon^{2}\right)\right)$. In this work, the spatial framework is used to study convective wake flow instabilities. Therefore, $\omega$ is real and linked with the circular frequency of an eigenmode $\tilde{\mathbf{q}}$, while $\alpha$ is the complex eigenvalue and its associated eigenvectors $\hat{\mathbf{q}}$ are to be sought. The real part $\alpha_{r}=\mathfrak{R}\{\alpha\}$ is related with the wavenumber of $\tilde{\mathbf{q}}$ along the homogeneous direction $x$ with wavelength $\lambda_{x}=2 \pi / \alpha_{r}$, whereas the imaginary part, $\alpha_{i}=$ $\mathfrak{J}\{\alpha\}$, is its growth/damping rate. A negative value of $\alpha_{i}$ stands for spatial growth of the eigenmode, while a positive value denotes spatial decay of $\tilde{\mathbf{q}}$. After discretization, a two-dimensional Generalized Eigenvalue Problem (GEVP) can be formed, but the resulting system is non-linear in eigenvalue $\alpha$. The companion matrix method $^{7}$ is used to convert the system into a linear eigenvalue problem by defining the auxiliary vector $\hat{\mathbf{q}}^{+}=[\hat{\rho}, \hat{u}, \hat{v}, \hat{w}, \hat{T}, \alpha \hat{u}, \alpha \hat{v}, \alpha \hat{w}, \alpha \hat{T}]^{T}$. The resulting two-dimensional GEVP is:

$$
\mathcal{A} \hat{\mathbf{q}}^{+}=\alpha \mathcal{B} \hat{\mathbf{q}}^{+}
$$

\subsubsection{Boundary conditions}

Adequate boundary conditions must be specified for the disturbance variables to solve the GEVP (Eqn. 3). No-slip, isothermal boundary conditions are imposed at the wall, i.e., $\hat{u}=\hat{v}=\hat{w}=\hat{T}=0$. In wall-normal direction, the perturbations are forced to decay by imposing homogeneous Dirichlet conditions at the upper boundary, which is placed far away from the region of interest to avoid reflections. The symmetry at the capsule zero meridian is exploited by imposing symmetric $\left(\hat{\rho}_{y}=\hat{u}_{y}=\hat{v}=\hat{w}_{y}=\hat{T}_{y}=0\right)$ and anti-symmetric $\left(\hat{\rho}=\hat{u}=\hat{v}_{y}=\hat{w}=\hat{T}=0\right)$ boundary conditions to reduce the computational effort. Corresponding Dirichlet conditions are set on the opposing side.

\subsubsection{Spatial discretization}

Equation 3 is solved on a grid independent from the one used for the basic flow computations. The wall-normal and spanwise directions are discretized with stable high-order finite differences schemes of order $q$ (FD- $q$ ), developed by Hermanns and Hernández. ${ }^{16}$ For the present study, the sixth-order scheme (FD- $q 6$ ) is used for both spatial directions. The number of discretization points was varied to ensure that the relevant flow quantities are insensitive to further improvement in grid resolution. ${ }^{23}$ All subsequent 2D spatial eigenvalue computations were performed with 89 points in spanwise and 101 points in wall-normal direction.

The interval of the non-uniform grid point distribution required by the FD- $q$ scheme $(\eta \in[-1,1])$ usually does not coincide with the physical domain of the problem studied (here: $\xi \in\left[0, \xi_{\infty}\right]$ with $\xi \hat{=} y, z$ ), therefore appropriate mappings need to be applied. In this work the same mapping function is chosen to cluster the points in the region of the roughness element wall normal extension and towards the symmetry plane

$$
\xi_{j}=l \frac{1-\eta_{j}}{1+b+\eta_{j}}, \quad b=2 l / \xi_{\infty}, \quad l=\frac{\xi_{\infty} \xi_{h}}{\xi_{\infty}-2 \xi_{h}},
$$

where $\xi_{\infty}$ denotes the location where the domain is truncated and $\xi_{h}$ represents the position where the number of discretization points is split into two halves. 


\section{Results}

In the following, results for the sphere-cone capsule will be presented for the flow cases summarized in Table 2. The roughness Reynolds numbers, $R e_{k k}$, as well as the slenderness ratio, $d / k$, are similar to the cases investigated with a cylindrical roughness element positioned in the leeward symmetry plane on the Apollo-shaped capsule (Cases 1-10 from Table 2 in Ref. 37) and corresponding results will also be shown again to facilitate comparisons. The differences in the laminar basic flow with regard to varying pressure gradients at equivalent roughness dimensions are addressed in the first subsection. In the second subsection, spatial 2D stability theory is used to work out the effect of roughness height on the instability properties, followed by considerations on the impact of the cylinder diameter in the last subsection.

Table 2: List of all investigated roughness dimensions.

\begin{tabular}{cccccccc}
\hline Case & $k[\mu \mathrm{m}]$ & $d[\mathrm{~mm}]$ & $d / k$ & $k / \delta$ & $k / \delta_{1}$ & $R e_{k k}$ & $M a_{k}$ \\
\hline 1 & 51.5 & 0.6866 & 13.33 & 0.094 & 0.587 & 150 & 0.49 \\
2 & 82.2 & 0.6324 & 7.69 & 0.150 & 0.937 & 348 & 0.75 \\
3 & 92.0 & 0.6134 & 6.67 & 0.168 & 1.048 & 424 & 0.83 \\
4 & 69.0 & 0.6580 & 9.54 & 0.126 & 0.786 & 254 & 0.64 \\
5 & 69.0 & 0.3450 & 5.00 & 0.126 & 0.786 & 254 & 0.64 \\
6 & 69.0 & 0.4600 & 6.67 & 0.126 & 0.786 & 254 & 0.64 \\
7 & 69.0 & 0.5750 & 8.33 & 0.126 & 0.786 & 254 & 0.64 \\
8 & 69.0 & 0.6900 & 10.00 & 0.126 & 0.786 & 254 & 0.64 \\
9 & 69.0 & 0.8050 & 11.67 & 0.126 & 0.786 & 254 & 0.64 \\
10 & 69.0 & 0.9200 & 9.54 & 0.126 & 0.786 & 254 & 0.64 \\
\hline
\end{tabular}

\subsection{Laminar basic flow}

Figure 6 illustrates the impact of a cylindrical element on the mean flow field for the two capsule configurations. At both investigated pressure gradients, the presence of the protuberance leads to a region of flow reversal both upstream and downstream of the roughness and to a formation of a counter-rotating vortex pair, known as the horseshoe vortex. The legs of the vortex bring down high-speed fluid from the outer region of the boundary layer towards the wall and transport low-speed fluid from the wall to the upper boundary layer region. This transport of momentum is known as the lift-up effect and generates streamwise velocity high and low-speed streaks, ${ }^{21}$ as depicted in Fig. 6 for different downstream positions $(s)$. The cylinder also induces a central low-speed streak due to the streamwise velocity blockage. The flow field along the capsule surface differs depending on the pressure gradient. The Mach number isocontours at $z=2 / 3 \mathrm{k}$ for the $50^{\circ}$-MSL capsule highlight the flow deceleration on the conical capsule part, whereas an almost constant acceleration occurs on the spherical forebody of the Apollo-shaped geometry. However, the spanwise profiles of streamwise velocity at selected locations behind the roughness trailing edge reveal a similar wake-flow development, though the amplitudes and spanwise gradients are more pronounced for the MSL capsule. Furthermore, both wake flows have in common, that due to the downwash motion of the vortices (high-momentum fluid from the upper boundary layer part is pushed towards the wall) the velocity deficit in the central low-speed streak region is first annihilated and then replaced by a high-speed streak. For the investigated different pressure gradients, the wake flow in the FPG case nearly relaxes towards the unperturbed state before it reaches the capsule shoulder, while for the APG condition a roughness-induced perturbation persists up to the end of the analyzed domain.

Figure 7 depicts the normalized wall-normal shear distribution in the symmetry plane at similar roughness Reynolds numbers $\left(50^{\circ}\right.$-MSL capsule: $R e_{k k}=348$, Apollo-shaped capsule: $\left.R e_{k k}=336\right)$ and identical slenderness ratio $(d / k=7.69)$. In addition, the solid black line indicates the spatial extent of the upstream and downstream reversed flow, revealing an upward tilt of the rearward recirculation zone in the APG case. For both pressure gradients, the intensity of the shear layer (originating at the roughness leading edge) decreases with downstream distance, whereas there is a second maximum in the wall-normal streamwise velocity gradient above the rear part of the reversed flow region on the $50^{\circ}$-MSL capsule, accompanied by an increase in wall-normal distance of the detached shear layer. In the FPG case, the vertical position of the shear layer remains nearly unchanged, probably due to a much smaller increase in boundary layer height compared to the APG condition.

The thickening of the boundary layer on the sphere-cone geometry is also depicted in Fig. 8 in terms of isolines of the streamwise velocity component in spanwise planes at three different downstream locations behind the cylindrical 


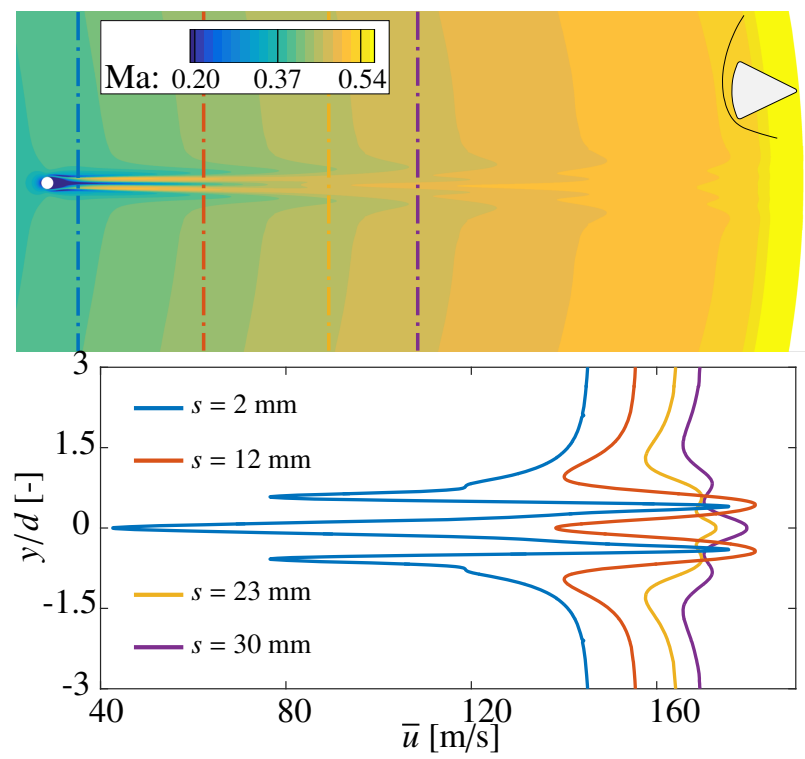

Apollo-shaped capsule, $R e_{k k}=336($ FPG case)

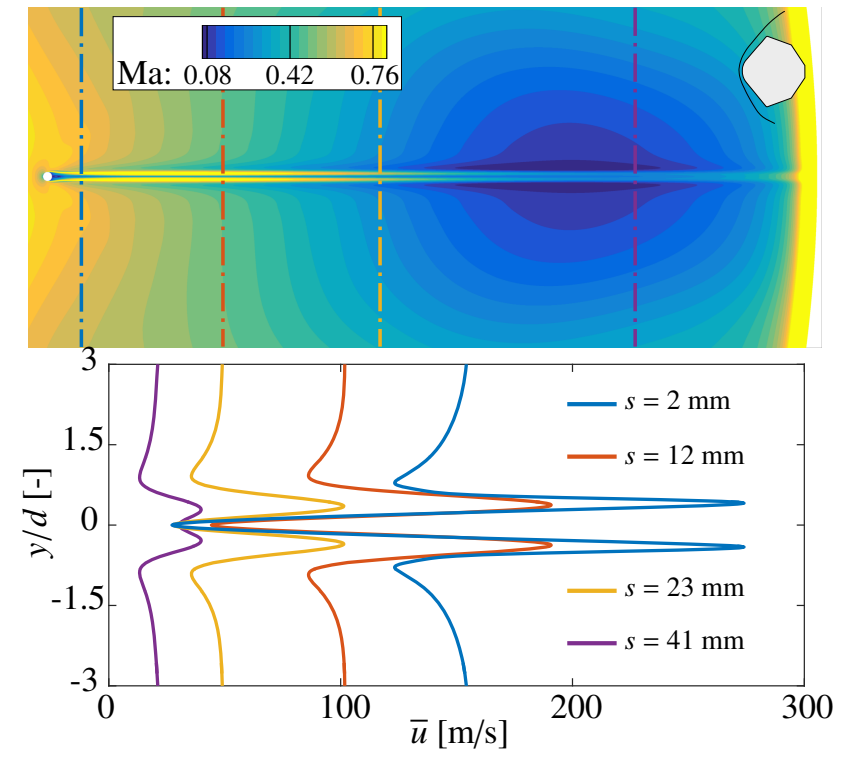

$50^{\circ}$-MSL capsule, $R e_{k k}=348(\mathrm{APG}$ case $)$

Figure 6: Mach number distribution (top row) and wake flow profiles of streamwise velocity (bottom row) for $d / k=$ 7.69 at $z=2 / 3 \mathrm{k}$.

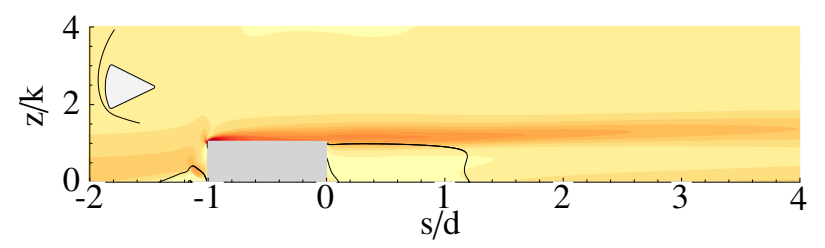

Apollo-shaped capsule, $R e_{k k}=336($ FPG case $)$

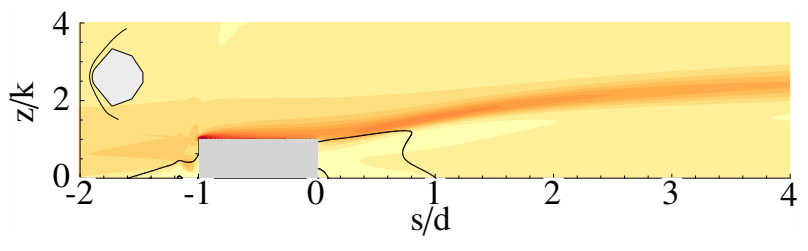

$50^{\circ}$-MSL capsule, $R e_{k k}=348($ APG case)

Figure 7: Normalized wall-normal shear $\partial \bar{u} / \partial z$ distribution in the symmetry plane around the roughness element with $d / k=7.69$. The reverse flow regions are visualized by black isolines of zero streamwise velocity.

roughness $\left(R e_{k k}=348\right)$. The intermediate to far wake flow region is characterized by the central low-speed streak with an upwelling of the flow, forming the typical mushroom-like structure, accompanied by an increase in boundarylayer thickness (compared to the unperturbed state). The central low-speed streak is surrounded by a pair of high-speed streaks with reduced boundary-layer thickness (solely one high-speed streak is visible since only one half of the plane is shown in Fig. 8). These streaks are characterized by high gradient regions, both in wall-normal and spanwise direction (see Fig. 8 top and bottom row).

\subsection{Roughness height variation}

A meaningful characteristic variable to quantify the impact of a discrete roughness element on the mean flow is the streak amplitude, ${ }^{3,10,37}$ defined as:

$$
A_{s t}=\frac{1}{2}\left[\max _{y, z}\left(\bar{u}-\overline{u_{b}}\right)-\min _{y, z}\left(\bar{u}-\overline{u_{b}}\right)\right],
$$

where $\bar{u}$ is the streamwise velocity and $\overline{u_{b}}$ denotes the undisturbed laminar basic flow. The evolution of the streak amplitude along the capsules symmetry meridians is shown in Fig. 9 for four different roughness Reynolds numbers. For both considered capsule geometries, the streak amplitude increases with roughness height and generally decreases with distance downstream of the roughness trailing edge in the far wake region $(s>5 \mathrm{~mm})$. For the roughness nearfield $\left(s<5 \mathrm{~mm}\right.$ ), a distinct increase in streak amplitude is present for the APG condition (and FPG with $\operatorname{Re}_{k k} \geq 278$ ), in the region where the main contribution on the streak amplitude shifts from the central low-speed streak towards the high-speed streak of the vortex system (see also Fig. 6). A substantial decrease of $A_{s t}$ for the sphere-cone shape occurs at $s \approx 34 \mathrm{~mm}$ where the flow starts to accelerate again towards the capsule shoulder (see also Fig. 6), further indicating 

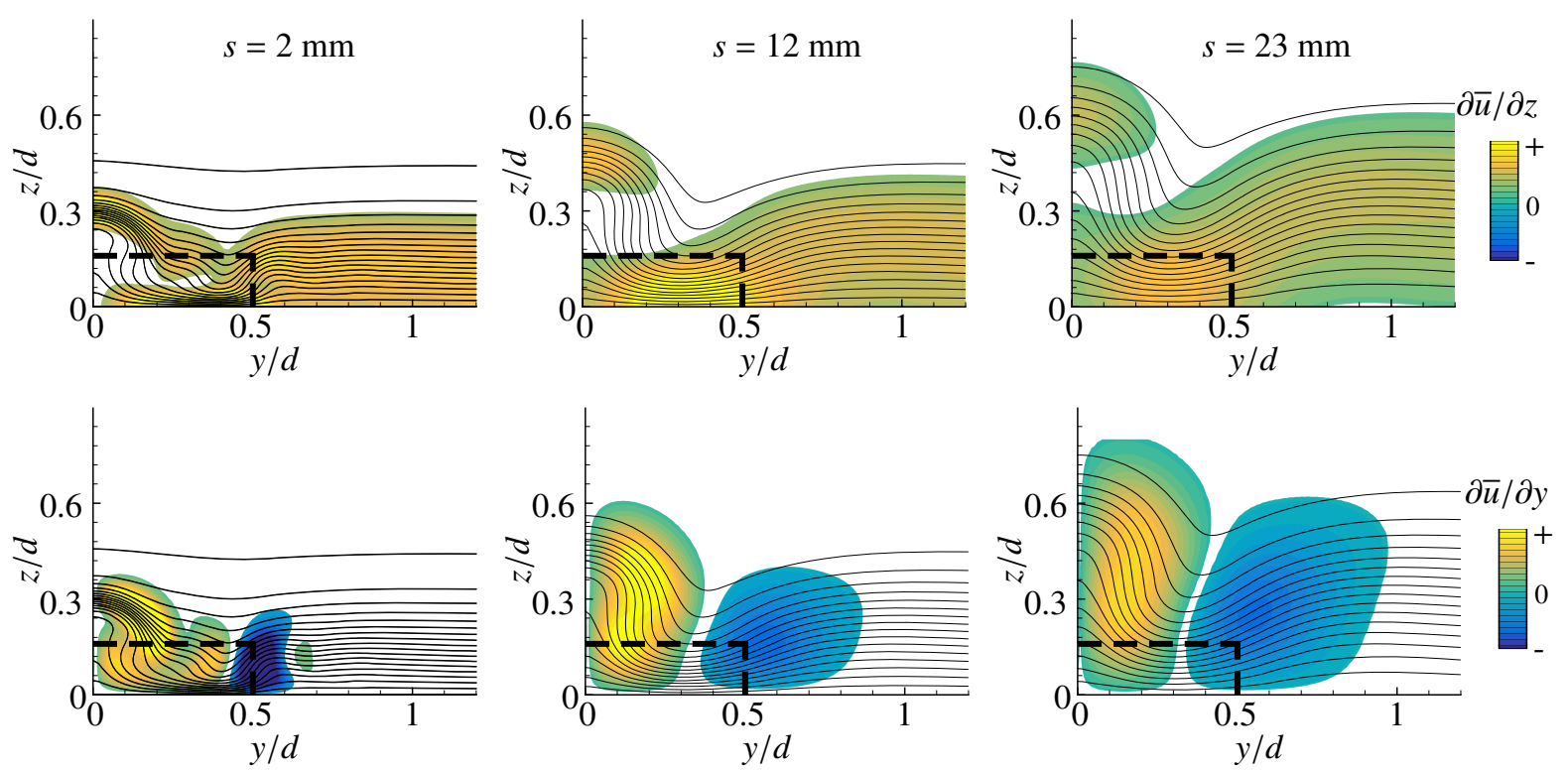

Figure 8: Spatial distribution of streamwise velocity wall-normal shear $\partial \bar{u} / \partial z$ (top row) and lateral shear $\partial \bar{u} / \partial y$ (bottom row) at three different planes behind the roughness element with $\operatorname{Re}_{k k}=348$ on the $50^{\circ}$-MSL capsule at $d / k=7.69$. The black isolines indicate the basic state $\bar{u}$-velocity distribution and the dashed line represents the projection of the roughness element. Shears below $10 \%$ of the absolute maximum are blanked out and due to the spanwise symmetry only the right half of the plane is shown.

the dampening effect of a favorable pressure gradient on roughness wake flows.

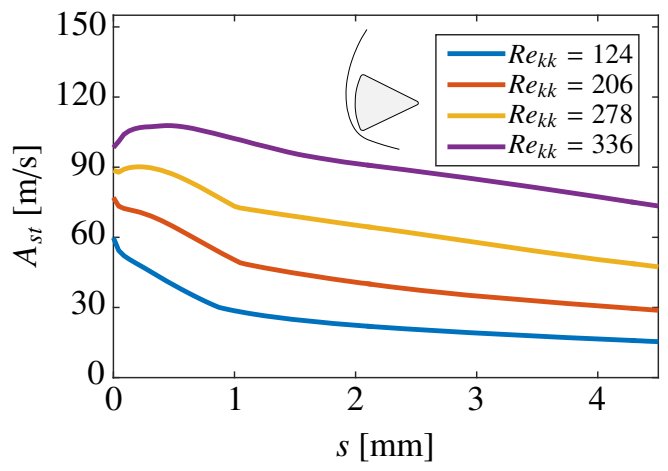

Apollo-shaped capsule (FPG case)

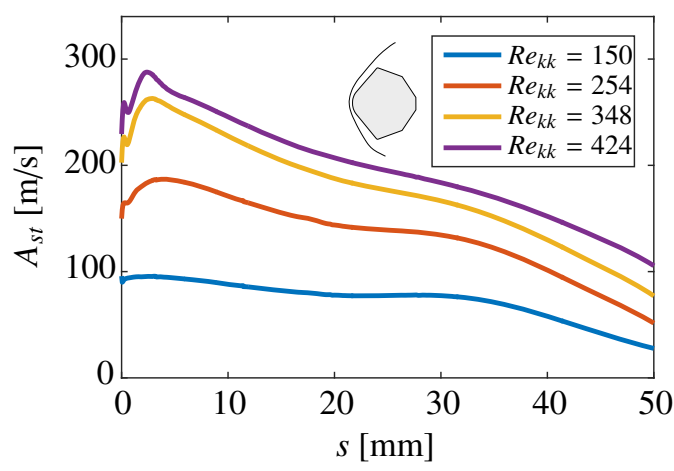

$50^{\circ}$-MSL capsule (APG case)

Figure 9: Evolution of streak amplitude along the capsule symmetry plane for various roughness Reynolds numbers. The position $s=0$ indicates the roughness trailing edge.

Two-dimensional spatial instability analyses for the stationary mean flow for all flow cases from Table 2 reveal both amplified symmetric modes (also known as varicose mode) and amplified anti-symmetric modes (sinuous mode) with respect to the streamwise velocity fluctuation. In the following, results will only be presented for the most amplified symmetric mode (S1), in terms of maximum $\mathrm{N}$-factor reached, and also for the most amplified anti-symmetric mode (A1). The integrated amplification rate, known as $\mathrm{N}$-factor, is defined as

$$
\mathrm{N}=\int_{s_{0}}^{s}-\alpha_{i} d s,
$$

where $s_{0}$ denotes the streamwise position where a disturbance starts to grow at a certain frequency.

The normalized mode shapes of the streamwise velocity fluctuation for the investigated dominant wake modes are visualized in Fig. 10 in a plane at $s=2 \mathrm{~mm}$ and at the peak disturbance frequency leading to the highest $\mathrm{N}$-factor, respectively. Note that the absolute value of the complex-valued disturbance amplitude functions is plotted. Although 

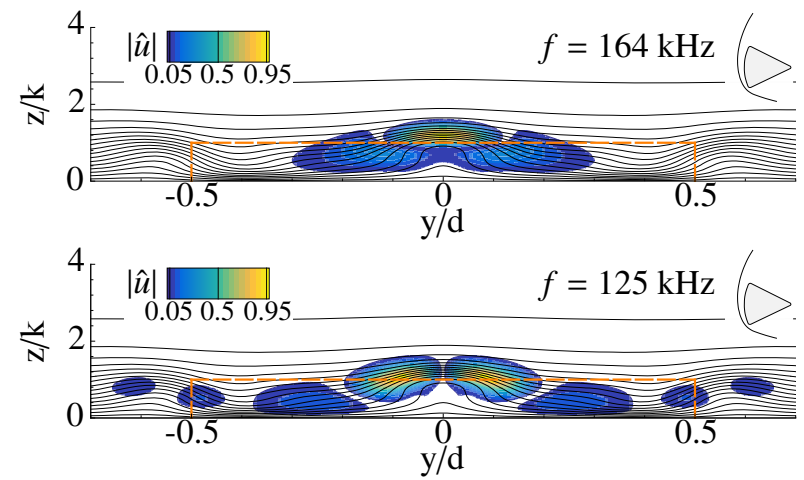

Apollo-shaped capsule, $R e_{k k}=336($ FPG case)
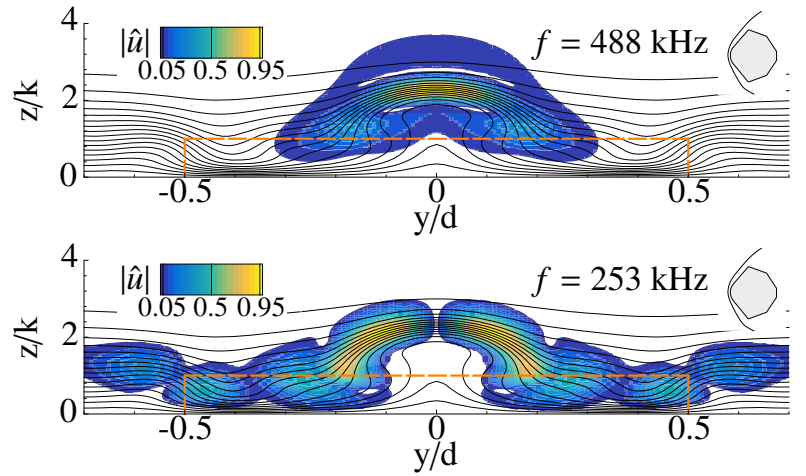

$50^{\circ}$-MSL capsule, $R e_{k k}=348($ APG case $)$

Figure 10: Normalized amplitude functions of the most amplified varicose (top row) and sinuous mode (bottom row) for the peak disturbance frequency at $s=2 \mathrm{~mm}$ and $d / k=7.69$. The black isolines indicate the basic state $\bar{u}$-velocity distribution and the dashed line represents the projection of the roughness element. Values $|\hat{u}|<0.05$ are blanked out.

the magnitude of the streamwise velocity amplitude function of the anti-symmetric modes appears to be symmetric, the corresponding fluctuations are opposite in phase. The velocity perturbation of the symmetric mode is concentrated near the top of the mushroom-like structure in a region with increased wall-normal shear $(\partial \bar{u} / \partial z$, see the upper row of Fig. 8). On the other hand, the anti-symmetric mode peaks on either side of the mushroom structure where the spanwise shear $(\partial \bar{u} / \partial y)$ is large (see the lower row of Fig. 8). The amplitude functions of the dominant wake modes display strong similarities, though the roughness-induced mean flow distortion is more pronounced in the case of an APG, associated with a shift of the shear layers in wall-normal direction (see also Fig. 7).

Because of the downstream recirculation zone behind the roughness and the formation of streamwise vortices, the wall-normal velocity component is included in the LST-2D for all sphere-cone cases. Figure 11 shows the N-factor envelope curves plotted versus the surface arc length for mode S1 (solid lines) and A1 (dashed lines) dependent on roughness Reynolds number. Note that the wall-normal velocity component had to be excluded for the production of mode S1 results on the Apollo capsule to prevent the avoided crossing phenomenon. ${ }^{37}$ Nevertheless, deviations in local growth rate due to wall-normal velocity influences are in the order of $10 \%$ and do not alter the trends presented in Fig. 11. For all roughness heights and both gradients considered, the dominant wake modes are already amplified at the first evaluation plane (similar to observations made by Choudhari et al. ${ }^{10,11}$ ) and the maximum $\mathrm{N}$-factor increases with roughness height. In the case of a favorable pressure gradient, the symmetric mode S1 leads to the highest $\mathrm{N}$-factor for all considered roughness Reynolds numbers, whereas there is no clear trend in the APG results. The maximum $\mathrm{N}$-factor of mode A1 for the intermediate roughness Reynolds numbers $\left(R e_{k k}=254\right.$ and $\left.R e_{k k}=348\right)$ is higher compared to the equivalent results for mode S1, while for the smallest and highest protrusion height the trend is inverted. The impact of a streamwise pressure gradient on the instability characteristics changes with the roughness Reynolds number. For the lowest value studied (Apollo-shaped capsule: $R e_{k k}=124,50^{\circ}$-MSL capsule: $R e_{k k}=150$ ), the maximum $\mathrm{N}$-factors on the spherical forebody as well as on the sphere-cone shape are about one for the dominant varicose mode. However, with increasing protrusion height modes S1 and A1 are more amplified at APG conditions. The peak $\mathrm{N}$-factor for either mode type is significantly larger for $R e_{k k}>150$, so an earlier laminar-turbulent transition downstream of the cylinder on the $50^{\circ}$-MSL capsule is expected for the investigated freestream conditions. On the other hand, higher roughness Reynolds numbers are needed to trip the boundary layer from a laminar to turbulent state on the Apollo-shaped capsule. For both forebody shapes considered, the dominant varicose mode S1 grows the fastest and may dominate the roughness-induced transition process, at least for $\operatorname{Re}_{k k}>254$ and when the initial amplitudes of both mode families are comparable. The spatial extent of the amplified wake flow region is significantly longer in an environment with a positive pressure gradient. The sinuous mode in the FPG case is amplified the furthest downstream, except for $R_{k k}=336$ where the amplitude of mode A1 grows up to about twice the amplified distance of the symmetric mode. In contrast, the dominant sinuous mode at APG conditions is always amplified the furthest downstream.

The bottom row of Fig. 11 shows the maximum N-factor computed downstream of the roughness at its corresponding frequency. For both investigated pressure gradients, the peak disturbance frequencies are higher for mode S1 in comparison to mode A1. This observation is in qualitative agreement with the findings of Choudhari et al. ${ }^{10}$ With increasing roughness Reynolds number, a broadening of the amplified frequency band occurs and the maximum $\mathrm{N}$-factor appears at higher frequencies (except for mode $\mathrm{A} 1$ at $R e_{k k}=336$ on the Apollo-shaped model). Figure 12 displays the most amplified disturbance frequency in terms of the non-dimensional frequency $F=f k / u_{k}$ for the investigated 

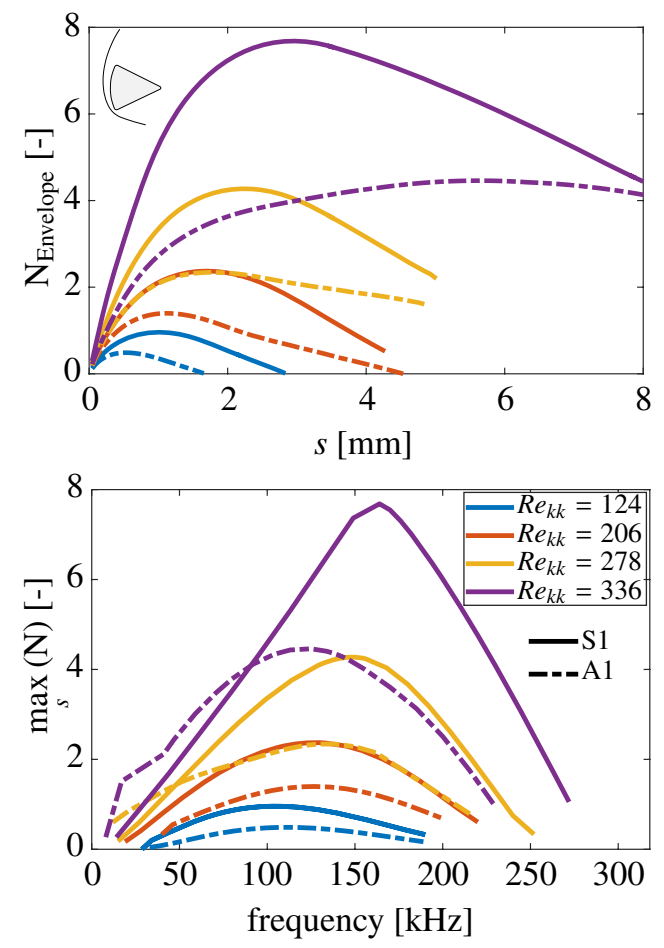

Apollo-shaped capsule (FPG case)
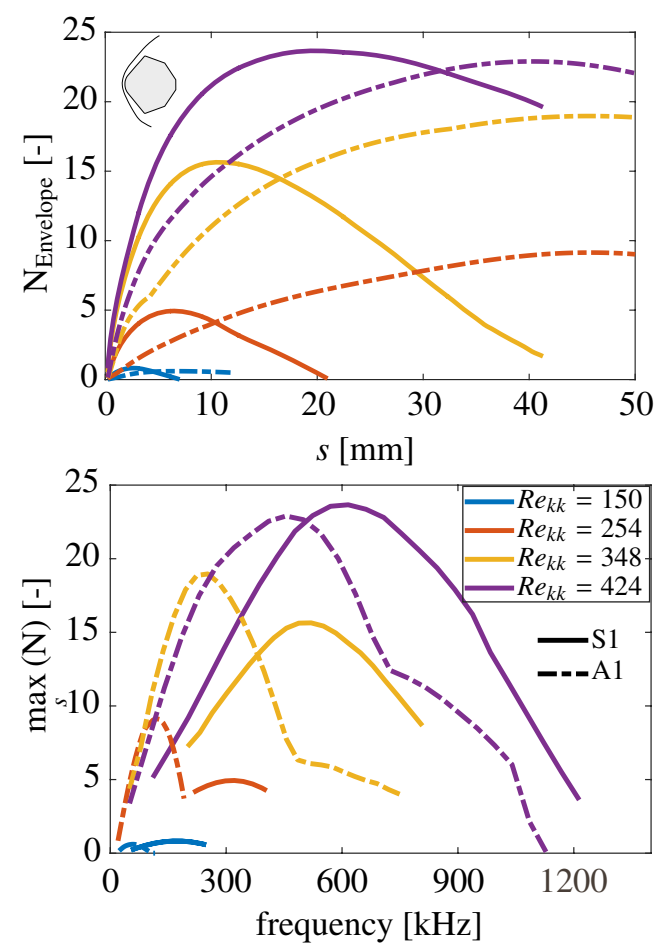

$50^{\circ}$-MSL capsule (APG case)

Figure 11: N-factor envelope curves versus surface arc length for the most amplified wake modes (top row) and maximum $\mathrm{N}$-factor as a function of disturbance frequency (bottom row) for all investigated roughness Reynolds numbers.

roughness Reynolds numbers. A linear-like dependency of $F$ from $R e_{k k}$ exists for the dominant varicose mode in both FPG and APG conditions. On the other hand, no clear trend is observable for the most amplified sinuous mode (not even if a reference length associated with the spanwise extent, such as $d$, is chosen). Hybrid modes on the sphere-cone geometry were not observed within this study, most likely due to a mismatch of the relevant frequency range (most unstable first-mode instability predicted at $f \approx 30 \mathrm{kHz}^{25,38}$ ).

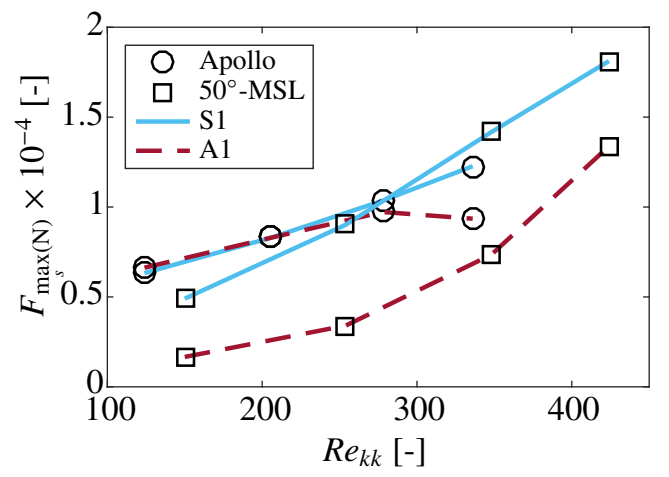

Figure 12: Peak non-dimensional disturbance frequency as a function of roughness Reynolds number.

To obtain a better insight into the mechanisms of how the shear layers of the basic flow transfer energy into the wake modes, a helpful method is to calculate the kinetic energy transfer in planes downstream of the roughness. ${ }^{6,26}$ The rate of change of kinetic energy is given by:

$$
\frac{\partial E_{K}}{\partial t}=\sum_{i=1}^{3} \sum_{j=1}^{3} P_{i, j}-\Phi, \quad P_{i, j}=\int_{z} \int_{y}-\tau_{i, j} \frac{\partial \overline{u_{i}}}{\partial x_{j}} d y d z, \quad \tau_{i, j}=\bar{\rho} \hat{u}_{i} \hat{u}_{j},
$$

where $E_{K}$ is the kinetic energy of the disturbance, $P_{i, j}$ the kinetic energy production term, $\Phi$ the viscous dissipation, and $\tau_{i, j}$ the Reynolds stress term. The sign of the production term $P_{i, j}$ indicates whether the local transfer of kinetic energy 
acts as stabilizing (negative sign) or destabilizing (positive sign) on the disturbance. Figure 13 shows the streamwise evolution of the kinetic energy production terms of mode S1 and A1 for the sphere-cone capsule at the peak disturbance frequency leading to the highest $\mathrm{N}$-factor, respectively. The basic flow shear layers related to the streamwise velocity component $\left(P_{1,1}, P_{1,2}, P_{1,3}\right)$ have the biggest contribution to the energy transfer, which is in accordance with the results obtained for the Apollo-shaped model ${ }^{37}$ (see inserts in Fig. 13). Depending on the streamwise pressure gradient, the relevance of the different production terms on the overall energy transfer varies within the unstable wake flow region (the end of the amplified region is indicated by the vertical dashed line in Fig. 13).

In the case of the favorable pressure gradient, mode S1 essentially extracts its energy from the work of the Reynolds stresses $\tau_{1,3}=\bar{\rho} \hat{u} \hat{w}$ against the wall-normal shear of the basic flow streamwise velocity component $\partial \bar{u} / \partial z$. This destabilizing effect of the wall-normal gradient diminishes as the central low-speed region starts to fade away, accompanied by the decrease in shear layer intensity (see Figs. 6 and 7). Once the counter-rotating vortex system establishes downstream of the element, $P_{1,2}$ has a positive contribution to the energy extraction process for both dominant wake modes. The production term $P_{1,1}$, related with the streamwise shear of the basic flow streamwise velocity component, stabilizes the dominant wake modes, especially in the vicinity of the reverse flow region.

In the case of the strong APG on the $50^{\circ}$-MSL capsule, the trend is reversed and $P_{1,1}$ destabilizes the dominant wake modes (it is only stabilizing when the flow begins to accelerate again towards the capsule shoulder). The kinetic energy production for mode S1 is no longer solely dominated by the $P_{1,3}$ term. Within the amplified wake flow region, a collaboration of mainly the $P_{1,3}$ term with the $P_{1,2}$ term is revealed, where $P_{1,3}$ has the biggest integral contribution to the overall energy production for the varicose mode and $P_{1,2}$ for the sinuous mode, respectively. The oscillation in the region $s=7-9 \mathrm{~mm}$ is caused by an interaction of expansion waves with the Dirichlet boundary condition at the upper boundary of the evaluation plane that is used in the instability analysis. Although the cylindrical roughness element is well submerged in the subsonic part of the boundary layer (see Fig. 4), the flow deflection above the roughness extends into the supersonic flow regime.

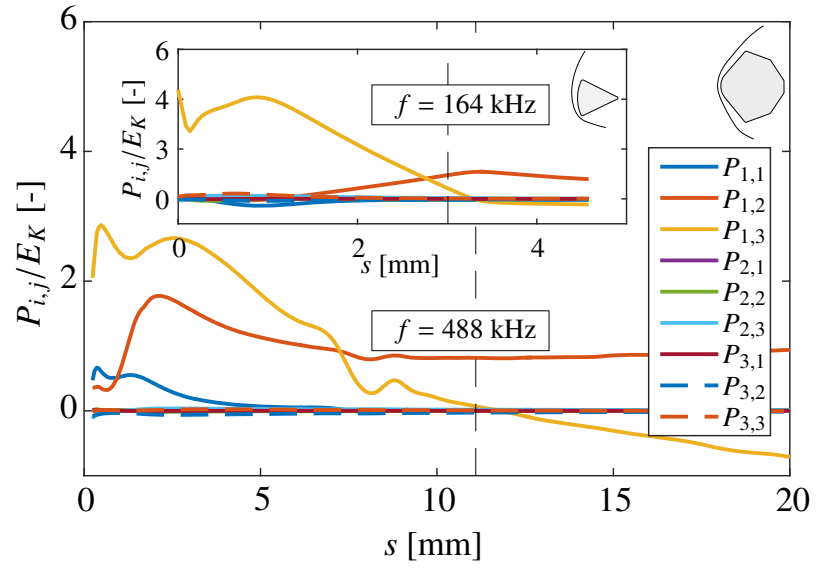

mode S1

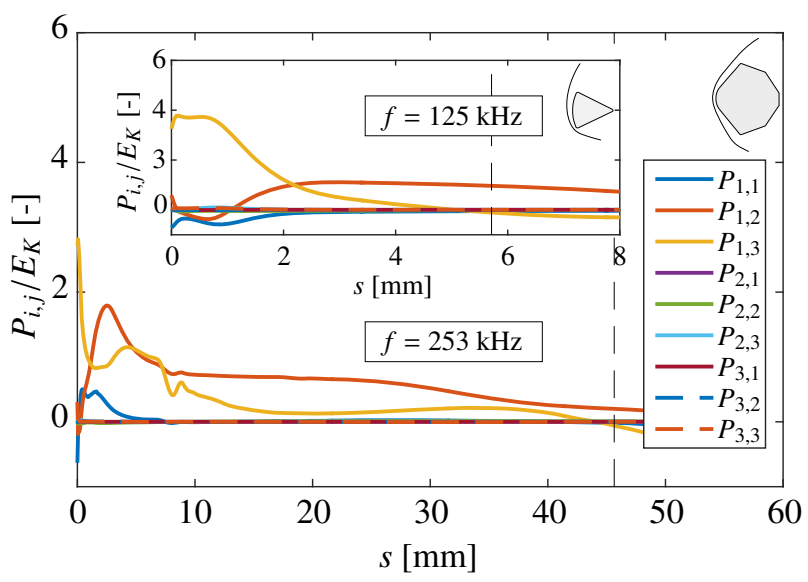

mode A1

Figure 13: Streamwise evolution of the kinetic energy production terms $P_{i, j}$ for mode S1 (left) and A1 (right) at $R e_{k k}=348$ for the $50^{\circ}$-MSL capsule. The black dashed line marks the downstream end of the amplified wake flow region for the indicated frequency. The inserts depict the equivalent results for the Apollo-shaped capsule at $R_{k k}=336$.

\subsection{Roughness diameter variation}

In this subsection results from a systematic variation of the roughness slenderness ratio, $d / k$, at a fixed roughness height will be presented. Hence, the roughness Reynolds number $R e_{k k}$ remains unchanged, although different $R e_{k k}$ values are used for the FPG and APG conditions. In our previous study for the Apollo-shaped capsule, the roughness width was varied at $R e_{k k}=336$. Depending on the roughness shape and slenderness ratio, avoided crossing occurred in the vicinity of the roughness trailing edge, which could be an indication of the existence of an absolute instability. Furthermore, the results from the previous subsection revealed a significant increase in the cumulative amplification ratios at APG conditions. Therefore, a value of $R e_{k k}=254$ is chosen for the present study to study only convective instabilities and to work out the effect of different roughness widths on wake mode instabilities subjected to a positive streamwise pressure gradient.

Figure 14 depicts the effect of different slenderness ratios on the evolution of the streak amplitude. For both 
pressure gradients considered, the roughness induced perturbation decreases with increasing protuberance width for the roughness nearfield. However, the streak amplitude linearly reduces for $s>1 \mathrm{~mm}$ with increasing downstream distance at FPG conditions, whereas in the APG case a second maximum evolves with increasing $d / k$ followed by a subsequent decrease in the region of flow acceleration. As a measure of roughness-induced perturbation, the evolution of the streak amplitude along the sphere-cone leeward line of symmetry indicates that with increasing roughness width, the amplified wake flow instabilities will likely need a longer distance to decay.

The instability results presented in Fig. 15 substantiate this hypothesis. The spatial extent of the amplified wake flow region increases with $d / k$ for both the most amplified varicose mode and the dominant sinuous mode at APG conditions, whereby the unstable region of mode A1 is about five times as long in comparison to the one of mode S1. In addition, the maximum $\mathrm{N}$-factor reached for mode $\mathrm{A} 1$ also increases with the slenderness ratio (for the APG case), revealing a strong correlation with the streak amplitude for the far-wake region (both APG and FPG). The dominant varicose mode is known to be linked with the shear layers along the central low-speed region ${ }^{26,37}$ (see also Fig.10) and as soon as the destabilizing effect of the wall-normal gradient diminishes (see evolution of $P_{1,3}$ term in Fig. 13), mode S1 becomes damped. The intensity of the detached shear layer decreases with roughness width (see e.g. Fig. 22 in Ref. 37), resulting in lower N-factors for mode S1 at both FPG and APG conditions. On the other hand, the vortex cores of the horseshoe vortex are further apart, leading to a slower reduction of the velocity deficit in the central low-speed streak region (accompanied by a slower reduction of the wall-normal shear) and therefore supposedly to the longer spatial extent of the amplified wake flow region of mode S1 (both APG and FPG).

The amplified disturbance frequency band shrinks with increasing slenderness ratio for both dominant wake modes on the sphere-cone model, as well as for mode S1 on the Apollo-shaped capsule (see the outer right plots in Fig. 15), but remains nearly unchanged for mode A1 (FPG condition). Furthermore, the most amplified disturbance frequency also reduces with roughness width and the relevant range of frequencies is essentially smaller for the sinuous mode. Although the roughness Reynolds number is different, the most unstable frequencies for the dominant antisymmetric mode are nearly the same at both investigated pressure gradients.

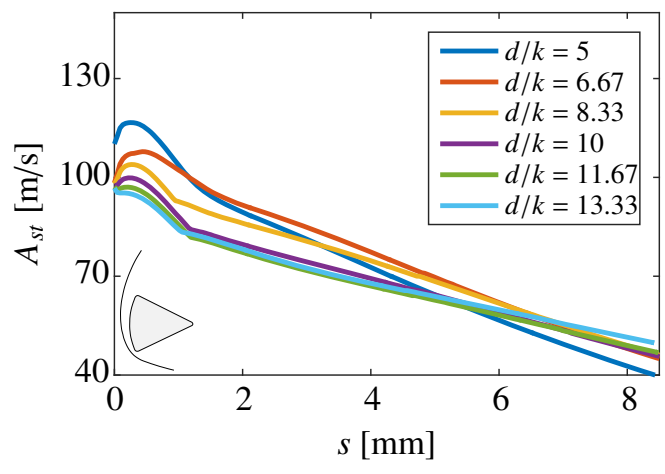

Apollo-shaped capsule, $R e_{k k}=336($ FPG case)

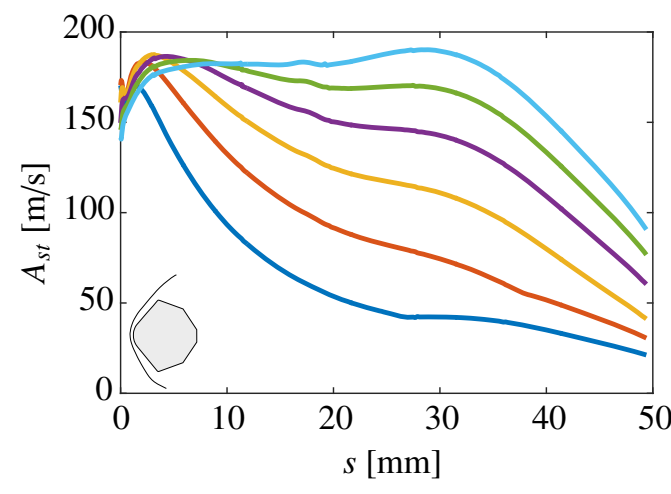

$50^{\circ}$-MSL capsule, $R e_{k k}=254($ APG case)

Figure 14: Evolution of streak amplitude along the capsule symmetry plane at various roughness slenderness ratios.

\section{Summary and concluding remarks}

Laminar steady basic flow computations were performed for a discrete cylindrical roughness element submerged in the boundary layer along the leeward symmetry plane of both an Apollo-like capsule and a MSL-like sphere-cone geometry at cold hypersonic wind tunnel conditions and at an angle of attack, respectively. Starting from the stagnation point, the flow on the Apollo capsule accelerates towards the shoulder, exhibiting a favorable pressure gradient, whereas on the $50^{\circ}$-MSL capsule, the flow is at first strongly accelerated over the spherical part followed by a distinct flow deceleration on the conical part. The dimensions of the roughness element were varied in both height and diameter to study the roughness wake flow development and the inherent instabilities subjected to different streamwise pressure gradients. The flow topology around the protuberance revealed a strong resemblance at both pressure gradients considered. Though, the roughness induced shear layers are longer preserved in an adverse pressure gradient (APG) environment. The streak amplitude, which is a measure of the perturbation caused by the surface inhomogeneity, increases with roughness height and reduces with the cylinder diameter for the roughness nearfield. For all investigated roughness dimensions, the streak amplitude decays towards the shoulder in case of a favorable streamwise pressure gradient (FPG). However, depending on the roughness Reynolds number $\left(R e_{k k}\right)$ and the slenderness ratio $(d / k)$, an additional maximum 

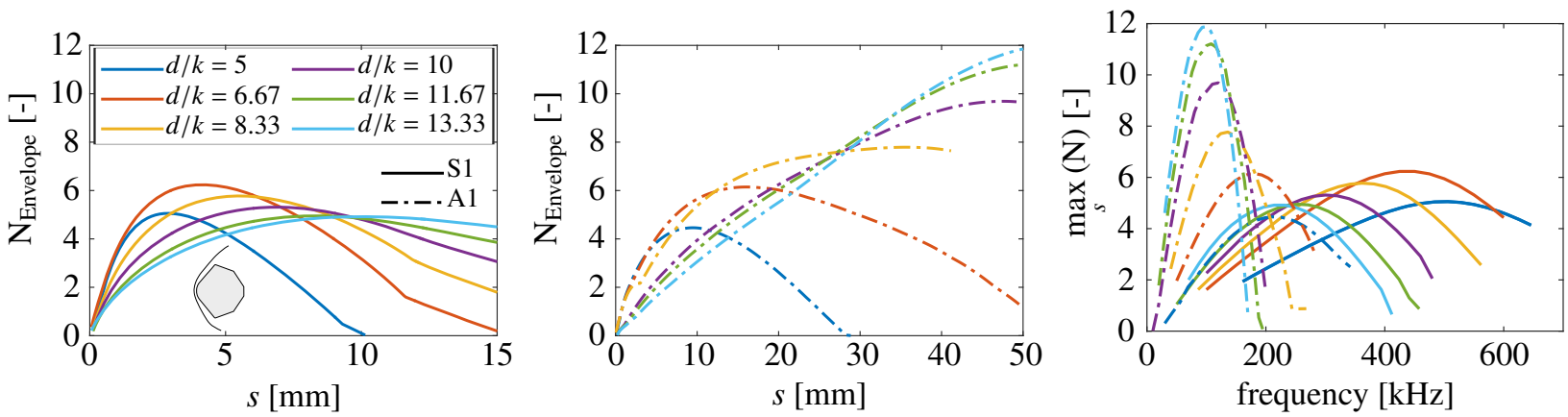

$50^{\circ}$-MSL capsule, $R e_{k k}=254($ APG case)
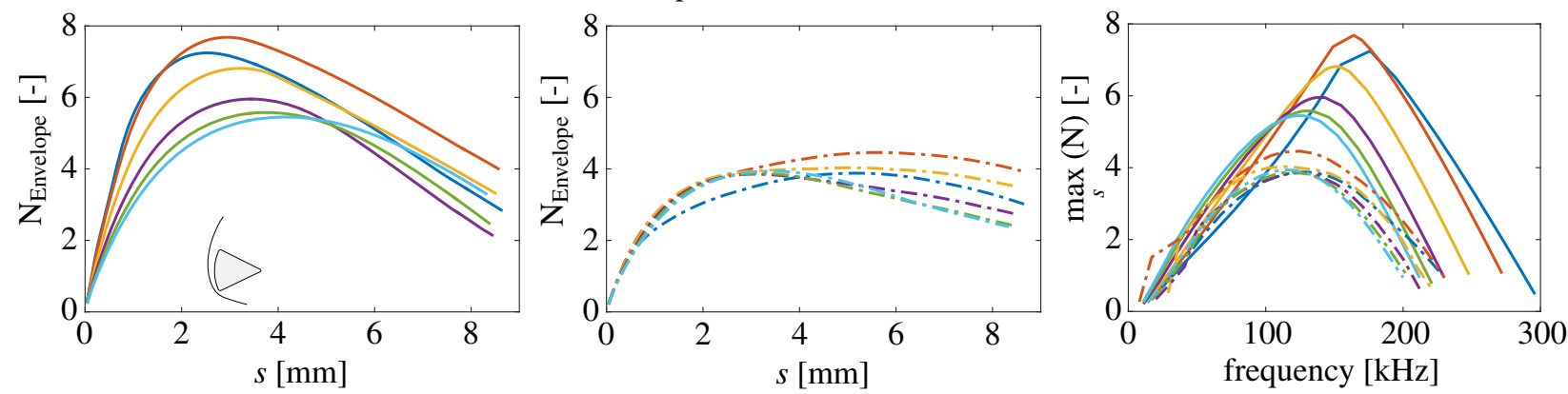

Apollo-shaped capsule, $R e_{k k}=336$ (FPG case)

Figure 15: N-factor envelope curves versus surface arc lengths for the most amplified varicose mode (left column), sinuous mode (middle column), and maximum obtained $\mathrm{N}$-factor as a function of disturbance frequency (right column) for all investigated slenderness ratios.

evolves far downstream of the element at APG conditions. Once the flow starts to accelerate again towards the shoulder on the $50^{\circ}$-MSL capsule, the streak amplitude substantially decreases due to the upcoming negative pressure gradient.

The instability properties of the streaky wake flow behind the cylinder were analyzed with the help of spatial two-dimensional instability theory (LST-2D), where the focus was on the most amplified symmetric (varicose) and anti-symmetric (sinuous) modes. It turned out that on the sphere-cone capsule (APG) the unstable wake flow region extends much further downstream and the wake mode instabilities are considerably more amplified in terms of modal $\mathrm{N}$-factor data. For all considered roughness dimensions, the most amplified sinuous mode (A1) is the one that is always amplified the furthest downstream in the APG region, whereas in the FPG case this only holds for the highest roughness Reynolds number investigated $\left(R e_{k k}=336\right)$. However, the amplitude of the most amplified symmetric mode (S1) grows the fastest (both in FPG and APG) and will most likely dominate the roughness-induced transition process above a critical threshold value, which has to be a function of height, planform shape, slenderness ratio, pressure gradient, and wall temperature. On the other hand, mode A1 grows slower in the beginning (both at FPG and APG conditions), but can reach higher $\mathrm{N}$-factors downstream (only valid for the APG case).

An analysis of the disturbance kinetic energy production terms revealed, that the basic flow shear layers related to the streamwise velocity component have the biggest contribution to the energy transfer. In particular, the wall-normal shear layer, formed at the roughness leading edge, solely destabilizes mode S1 on the Apollo-shaped capsule, whereas mode S1 and A1 both originate from instability of the whole three-dimensional shear layer at the investigated APG conditions. Nonetheless, the varicose mode is mainly linked with the wall-normal shear, whereas the lateral shear has the biggest integral contribution to the energy transfer for the sinuous mode. The favorable pressure gradient reduces the growth of the dominant wake modes, whereas an adverse pressure gradient is destabilizing the wake.

\section{Acknowledgments}

Funding of this work by the German Research Foundation (DFG) grant HE 5440/4-2 is gratefully acknowledged. 


\section{References}

[1] S. R. C. Ali, R. Radespiel, and A. Theiss. Transition experiment with a blunt Apollo shape like capsule in hypersonic Ludwieg tube. 63. Deutscher Luft- und Raumfahrtkongress 2014, Paper 2014-340270, 2014.

[2] A. Amar, T. Horvath, B. Hollis, K. Berger, S. Berry, and N. Calvert. Protuberance boundary layer transition for project orion crew entry vehicle. Paper 2008-1227, AIAA, 2008.

[3] P. Andersson, L. Brandt, A. Bottaro, and D. S. Henningson. On the breakdown of boundary layer streaks. J. Fluid Mech., 428:29-60, 2001.

[4] K. T. Berger. Aerothermodynamic testing of the Crew Exploration Vehicle at Mach 6 and Mach 10. J Spacecr Rockets, 46(4):758-765, 2009.

[5] M. Bernardini, S. Pirozzoli, P. Orlandi, and S. K. Lele. Parameterization of boundary-layer transition induced by isolated roughness elements. AIAA Journal, 52(10):2261-2269, 2014.

[6] L. Brandt. Numerical studies of the instability and breakdown of a boundary-layer low-speed streak. Eur. J. Mech. B. Fluids, 26(1):64-82, 2007.

[7] T. Bridges and P. Morris. Differential eigenvalue problems in which the parameter appears nonlinearly. J. Comput. Phys., 55(3):437-460, 1984.

[8] C.-L. Chang, M. Choudhari, B. Hollis, and F. Li. Transition analysis for the Mars Science Laboratory entry vehicle. Paper 2009-4076, AIAA, 2009.

[9] C.-L. Chang, M. Choudhari, B. S. Venkatachari, and F. Li. Effects of cavities and protuberances on transition over hypersonic vehicles. Paper 2011-3245, AIAA, 2011.

[10] M. Choudhari, F. Li, C.-L. Chang, J. Edwards, M. Kegerise, and R. King. Laminar-turbulent transition behind discrete roughness elements in a high-speed boundary layer. Paper 2010-1575, AIAA, 2010.

[11] M. Choudhari, A. Norris, F. Li, C.-L. Chang, and J. Edwards. Wake instabilities behind discrete roughness elements in high speed boundary layers. Paper 2013-81, AIAA, 2013.

[12] M. M. Choudhari, F. Li, M. D. Bynum, M. A. Kegerise, and R. A. King. Computations of disturbance amplification behind isolated roughness elements and comparison with measurements. Paper 2015-2625, AIAA, 2015 .

[13] J. P. J. P. V. den Eynde and N. D. Sandham. Numerical simulations of transition due to isolated roughness elements at Mach 6. AIAA Journal, 54(1):53-65, 2016.

[14] G. Groskopf and M. J. Kloker. Instability and transition mechanisms induced by skewed roughness elements in a high-speed laminar boundary layer. J. Fluid Mech., 805:262-302, 2016.

[15] G. Grossir, S. Paris, and O. Chazot. Evaluation of the effects of distributed roughness induced transition on the EXPERT vehicle. In International Planetary Probe Workshop 2010 (IPPW-7).

[16] M. Hermanns and J. A. Hernández. Stable high-order finite-difference methods based on non-uniform grid point distributions. Int. J. Numer. Methods Fluids, 56(3):233-255, 2007.

[17] B. Hollis, D. Liechty, M. Wright, M. Holden, T. Wadhams, M. MacLean, and A. Dyakonov. Transition onset and turbulent heating measurements for the Mars Science Laboratory entry vehicle. Paper 2005-1437, AIAA, 2005.

[18] B. R. Hollis. Distributed roughness effects on blunt-body transition and turbulent heating. Paper 2014-0238, AIAA, 2014.

[19] P. S. Iyer and K. Mahesh. High-speed boundary-layer transition induced by a discrete roughness element. J. Fluid Mech., 729:524-562, 2013.

[20] M. A. Kegerise, R. A. King, M. Choudhari, F. Li, and A. T. Norris. An experimental study of roughness-induced instabilities in a supersonic boundary layer. Paper 2014-2501, AIAA, 2014.

[21] M. T. Landahl. On sublayer streaks. J. Fluid Mech., 212(1):593-614, 1990. 
[22] A. N. Leidy, E. Reshotko, F. Siddiqui, and R. D. W. Bowersox. Characterizing the transient growth mechanism on a hypersonic blunt body at a high angle of attack. Paper 2016-3951, AIAA, 2016.

[23] S. Leyh. Numerische Stabilitätsuntersuchung des Nachlaufs einer diskreten Rauigkeit auf einer Wiedereintrittskapsel. Master's thesis, Technische Universität Berlin, 2017.

[24] F. Li, M. Choudhari, C.-L. Chang, and J. White. Effects of injection on the instability of boundary layers over hypersonic configurations. Phys. Fluids, 25(10):104-107-1-15, 2013.

[25] M. Lichtmes. Numerische Untersuchung zur Grenzschichtstabilität an der Atmosphäreneintrittskapsel des Mars Science Laboratory im hypersonischen Strömungsfeld. Master's thesis, Hochschule Trier - Umwelt-Campus Birkenfeld, 2015.

[26] J.-C. Loiseau, J.-C. Robinet, S. Cherubini, and E. Leriche. Investigation of the roughness-induced transition: global stability analyses and direct numerical simulations. J. Fluid Mech., 760:175-211, 2014.

[27] E. Marineau, S. Laurence, and H. Hornung. Apollo-shaped capsule boundary layer transition at high-enthalpy in T5. Paper 2010-446, AIAA, 2010.

[28] O. Marxen, G. Iaccarino, and E. Shaqfeh. Numerical simulations of hypersonic boundary-layer instability with localized roughness. Paper 2011-567, AIAA, 2011.

[29] B. Plogmann, W. Würz, and E. Krämer. On the disturbance evolution downstream of a cylindrical roughness element. J. Fluid Mech., 758:238-286, 2014.

[30] J. Raddatz and J. K. Fassbender. Block structured navier-stokes solver FLOWer. In MEGAFLOW - Numerical Flow Simulation for Aircraft Design, pages 27-44. Springer Berlin Heidelberg, 2005.

[31] D. C. Reda. Review and synthesis of roughness-dominated transition correlations for reentry applications. $J$ Spacecr Rockets, 39(2):161-167, 2002.

[32] D. C. Reda, M. C. Wilder, and D. K. Prabhu. Transition experiments on blunt bodies with isolated roughness elements in hypersonic flight. J Spacecr Rockets, 47(5):828-835, 2010.

[33] S. P. Schneider. Summary of hypersonic boundary-layer transition experiments on blunt bodies with roughness. J Spacecr Rockets, 45(6):1090-1105, 2008.

[34] H. Tanno, T. Komuro, K. Sato, K. Itoh, R. P. Lillard, J. Olejniczak, and J. H. Grinstead. Aeroheating measurement of Apollo shaped capsule with boundary layer trip in the free-piston shock tunnel HIEST. Paper 2014-0434, AIAA, 2014.

[35] A. Theiss, S. R. Ali, S. Hein, D. Heitmann, and R. Radespiel. Numerical and experimental investigation of laminar-turbulent boundary layer transition on a blunt generic re-entry capsule. Paper 2014-2353, AIAA, 2014.

[36] A. Theiss and S. Hein. Investigation on the wake flow instability behind isolated roughness elements on the forebody of a blunt generic re-entry capsule. In 6th European Conference for Aeronautics and Space Sciences, 2015.

[37] A. Theiss, S. Hein, S. R. C. Ali, and R. Radespiel. Wake flow instability studies behind discrete roughness elements on a generic re-entry capsule. Paper 2016-4382, AIAA, 2016.

[38] A. Theiss, M. Lichtmes, and S. Hein. Local stability analysis of laminar-turbulent boundary layer transition on blunt generic re-entry capsules. In Notes on Numerical Fluid Mechanics and Multidisciplinary Design, pages 279-288. Springer International Publishing, 2016.

[39] N. D. Tullio, P. Paredes, N. D. Sandham, and V. Theofilis. Laminar-turbulent transition induced by a discrete roughness element in a supersonic boundary layer. J. Fluid Mech., 735:613-646, 2013.

[40] B. M. Wheaton and S. P. Schneider. Hypersonic boundary-layer instabilities due to near-critical roughness. J Spacecr Rockets, 51(1):327-342, 2014. 